\title{
Session 2: Cancer I
}

Monday 29th October 2007. Moderators: Zdenka Jonak and Mark Glassy

\author{
[11.00-11.30] \\ Reversible anergy of surface IgM-mediated \\ signaling in the two subsets of chronic lymphocytic \\ leukemia defined by immunoglobulin $\mathrm{V}_{H}$ \\ mutational status \\ Kathleen N Potter ${ }^{\mathrm{a}}$, C Ian Mockridge ${ }^{\mathrm{a}}$, Isla \\ Wheatley $^{\mathrm{a}}$, Louise A Neville ${ }^{\mathrm{a}}$, Graham Packham ${ }^{\mathrm{b}}$, \\ and Freda K Stevenson ${ }^{\text {a }}$ \\ ${ }^{a}$ Molecular Immunology Group, Tenovus Laboratory \\ ${ }^{\mathrm{b}}$ Cancer Research UK Clinical Centre, Cancer \\ Sciences Division, School of Medicine, University of \\ Southampton, Southampton General Hospital, \\ Southampton SO16 6YD, UK
}

Abstract: Chronic lymphocytic leukemia (CLL), a monoclonal $\mathrm{CD}^{+} \mathrm{B}$ cell disorder, is the most commonly occurring leukemia in the Western hemisphere. There are two subsets of CLL based on $\mathrm{V}_{H}$ mutational status; unmutated (U-CLL) cases generally have worse prognosis while mutated (M-CLL) cases have better prognosis. Previous studies indicated that the different clinical behavior could be related to the ability of the tumor cells to respond to surface immunoglobulin (sIg) mediated signals. U-CLL had an increased ability to phosphorylate $\mathrm{p} 72^{\text {Syk }}$ in response to $\mathrm{sIgM}$ ligation compared to M-CLL. We now confirm and further investigate this differential signalling in a large cohort by intracellular calcium mobilization. Cases responding to sIgM ligation express higher levels of CD38, ZAP70 and sIgM. However, CD38 does not influence signalling in vitro or associate with response in bimodal cases in which some of the tumor cells are $\mathrm{CD}_{3} 8^{+}$and within the same individual some of the tumor cells are CD38- ${ }^{-}$. Similarly, ZAP-70 expression is not required for a calcium response in either U-CLL or M-CLL. Strikingly, partially or completely anergized SIgM responses from each subset can recover both sIgM expression and signal capacity spontaneously in vitro or following endocytosis. The most likely explanation for this recovery is the in vitro removal of putative antigen which is engaging SIgM in vivo, although alternative events occurring in vitro cannot be discounted. Signaling via sIgD differs markedly from signalling via sIgM by being almost universally positive in both U-CLL and M-CLL, with no association with CD38 or ZAP70 expression. Downstream signalling pathways therefore appear intact in CLL, locating anergy to SIgM, and mainly in the M-CLL subset. Integration of differential isotype-specific effects mediated by (auto)antigen may determine tumor B cell behaviour in CLL.

\section{[11.30-11.50]}

'Antibody-based cancer therapy through targeting metastasis, angiogenesis and immunosuppression factors'

Yan $\mathrm{Wu}$

ImClone Systems Inc., New York, USA

The role of TGF $\beta$ signaling in regulating tumor progression has been widely documented. High level of TGF $\beta$ in tumor patient serum was reported to be correlated with highly metastatic phenotype and poor clinical outcome. TGF- $\beta$ signaling promotes metastasis via eliciting epithelial-to-mesenchymal transition (EMT) of cancer cells toward a highly invasive and metastatic tumor phenotype. TGF $\beta$ signaling also indirectly promote metastasis by increasing extracellular matrix production/degradation, mediating tumor stromal formation, and inducing tumor vascularization. The role of TGF $\beta$ signaling pathways in tumor-induced immunosuppression has been studied extensively in the past years. Blockade of TGF- $\beta$ signaling by the biological agents led to enhanced antitumor activity resulting from the suppression of metastasis and angiogenesis and a decrease in Treg cells-associated immunosuppression. Targeting TGF $\beta$ receptor by a specific mAb would be a viable approach to the inhibition of TGF $\beta$ signaling mediated cancer growth and progression. This report will discuss on the development of anti-TGF $\beta$ receptor antibodies and their antitumor activity in the 
inhibition of tumor metastasis and angiogenesis, and enhancement of antitumor immunity in in vitro and in vivo experimental models.

[11.50-12.10]

\section{'Targetting tumour vasculature using a novel antibody-IL-12 fusion protein' \\ Nigel Courtenay-Luck \\ Antisoma Research Ltd, London, UK}

Abstracts not provided.

[12.10-12.30]

Development of IMC-1121B, a fully human antibody directed against vascular endothelial growth factor receptor 2 for anti-angiogenic therapy

Zhenping Zhu

ImClone Systems Incorporated, New York, NY, USA

Tel.: +1646 638 5190; Fax: +1 212645 2054;

E-mail: zhenping.zhu@imclone.com

Compelling evidence indicate that uncontrolled angiogenesis play an important role in a variety of human pathological conditions, including cancer and retinopathy. Many attempts have been made to identify and develop effective angiogenesis inhibitors for therapeutic purposes. We produced a fully human, high affinity antibody directed against vascular endothelial growth factor (VEGF) receptor 2 (KDR), IMC-1121B, from an antibody phage display library. IMC-1121B binds to KDR with high affinity $(\sim 50 \mathrm{pM})$, and potently neutralizes a variety of VEGF-induced biological activities, including receptor activation and downstream signaling, and endothelial cell migration and proliferation. In animal models, IMC-1121B significantly prolonged the survival of NOD-SCID mice inoculated with $\mathrm{KDR}^{+}$ human leukemia cells. Further, IMC-1121B strongly blocked the development of laser-induced choroidal neovascularization (CNV) in a primate model. A phase I clinical trial of IMC-1121B has been completed in patients with advanced malignancies, and encouraging preliminary results have been reported.

\section{[12.30-12.50]}

'Adecatumumab, a fully human EpCAM antibody in anti-tumour immunotherapy'

Tobias Raum

Micromet AG, Munich, Germany

MT201 is a fully human antibody against the pancarcinoma Ep-CAM antigen. This antibody is derived from phage display guided selection using human antibody libraries. Its specificity proved to be efficatious in animal tumour models. The therapeutic potential of MT201 was further explored for the treatment of tumour patients. Results from clinical phases I and II in prostate and breast cancer will be presented. 